\title{
Correlation of Hematological Parameters and NESTROFT in A Routine Hematology Laboratory - A Prospective Study
}

\author{
Bheema Rao $\mathrm{G}^{1}$,Aishwarya $\mathrm{S}^{2}$,Priavadhana Rajan Prasaad ${ }^{3}$ \\ Department Of Pathology, Sree Balaji Medical College And Hospital, Chennai, Tamil Nadu, India. \\ ${ }^{1}$ Associate Professor, Department Of Pathology, SBMCH, Chennai \\ ${ }^{2}$ Post Graduate, Department Of Pathology, SBMCH, Chennai \\ ${ }^{3}$ Assistant Professor, Department Of Pathology, SBMCH, Chennai
}

\begin{abstract}
Thalassemia syndromes are considered to be the most common genetic disorders worldwide. Hence, prenatal diagnosis using genotyping techniques are designed for the early diagnosis and management. The costs of these prenatal diagnostic techniques as well as confirmatory tests for these thalassemic syndromes are expensive to be made available in poor resourced countries. Hence, screening tests were developed for field diagnosis of these disorders. Naked Eye Single Tube Red Cell Osmotic Fragility Test (NESTROFT) was designed as a simple screening test for the detection of thalassemic syndromes. In this study, we compared two groups which showed positivity for NESTROFT with the negative test group for all the haematological parameters available by an automated hematology analyzer so as to decide the samples to be tested for NESTROFT in a routine Clinical Pathology Laboratory.

Materials and Methods: All the peripheral smears and complete hemograms received in the clinical pathology laboratory during the period of July 2016 to December 2016 were included in the study. Peripheral smear findings and their corresponding haematological parameters were evaluated. NESTROFT was carried out in all the samples and the test results are compared with their corresponding peripheral smear findings and haematological parameters.

Results: It was observed that in both the NESTROFT positive and negative test groups, there was no significant difference in the haematological parameters. None of the parameters could be used to narrow down the sample size to be used for NESTROFT screening in the routine clinical pathology laboratory.

Conclusion: It is concluded from our study that all peripheral smears with a microcytic hypochromic anaemia especially in women of child bearing age group should be screened for thalassemia trait by using NESTROFT as a routine test in clinical laboratories.
\end{abstract}

Keywords: Osmotic fragility, Beta Thalassemia trait, Genotyping

\section{Introduction}

Thalassemia syndromes are considered to be the most common genetic disorders worldwide. It comprises of a heterogenous group of Mendelian disorders characterized by decreased or lack of synthesis of either the alpha-globin chains (alpha thalassemia) or the beta-globin chains (beta thalassemia) of haemoglobin. Worldwide, frequency of thalassemia trait is about $3 \%$, whereas in India the frequency ranges from $3-18 \%$. In few communities like Sindhis, Kutchis, Lohanas, Bhanusalis, Punjabis, Mahars, Agris, Gaud, Saraswats, Gowdas etc. in India, studies show that thalessemia trait is found to have a higher frequency.

Apart from haemoglobin electrophoresis, where consistently elevated HbA2levels are confirmatory, various other haematological investigations available are helpful in diagnosing beta thalassemia trait. However, these investigations are either expensive or time consuming or cumbersome and often require sophisticated equipment. Hence, they cannot be used as effective tools for population screening. For screening purposes, a test which is inexpensive, requires a small amount of blood, does not require sophisticated equipment and can be applied on the population as a whole is preferred. These requirements are met by a modified osmotic fragility test "NESTROFT" (Naked Eye Single Tube Red Cell Osmotic Fragility Test), a test first described by Kattamis et al. In this study, we compared two groups which showed positivity for NESTROFT with the negative test group for all the haematological parameters available by an automated hematology analyzer so as to decide the samples to be tested for NESTROFT in a routine clinical pathology laboratory.

\section{Materials and methods}

The present study was conducted in the department of haematology, Sree Balaji Medical College and Hospital in Chennai. The study includes patients of different age groups, attending the outpatient department of all the specialties presenting with various complaints associated with anemia. Complete 
haemogram and peripheral blood smear were done to confirm anemia and also to sub-type it. Subsequently, 50 cases who had a low haemoglobin $(\mathrm{Hb})$ value and diagnosed as microcytic hypochromic anaemia on peripheral blood smear are subjected to NESTROFT.

\section{Principle of nestroft:}

Normally, red cells put in saline solution begin to lyse at a saline concentration of $0.4-0.5 \%$ and lysis is complete at $0.32 \%$. However, in beta thalssemia trait, due to alteration in osmotic resistance of the affected RBC's due to volume/surface area ratio changes ${ }^{[4]}$ lysis begins at a saline concentration between $0.4-0.35 \%$ and it may not be completed even at $0.1 \%$ solution. NESTROFT is done at a saline concentration of $0.36 \%$ to ensure a high sensitivity with acceptable specificity. ${ }^{[1]}$

\section{Material}

$0.36 \%$ buffered saline prepared by diluting $36 \mathrm{ml}$ of $1 \%$ buffered saline with $64 \mathrm{ml}$ of distilled water to make $100 \mathrm{ml}$ (Test Reagent).

\section{Procedure of the test:}

Two test tubes labelled as ' $\mathrm{C}$ ' (control) consists of $2 \mathrm{ml}$ of distilled water and as ' $\mathrm{T}$ ' (test) consists of $2 \mathrm{ml}$ of $0.36 \%$ buffered saline. A drop of blood was added to each of the tubes, which were then left undisturbed for half an hour at room temperature. Following this, contents of both tubes were gently shaken and held against a white paper on which a thin black line was drawn. The line was clearly visible through the control tube and if it was the same in test tube; it was considered negative, otherwise test result was interpreted as positive [Fig-1,2]. The tubes were left undisturbed for 3 hours. At the end of 3 hours, the control tube was seen to be homogeneously pink with no sediments. In the 'test' tube the negative test showed similar findings as control tube where as in a positive case, a clear supernatant and a sediment at bottom was observed ${ }^{[5]}$. The observations collected from the NESTROFT test were recorded.

\section{Observations And Results}

The study population included 50 patients' hemograms and peripheral smears. The age range of this study population varied from one year to 58 years. All the patients had peripheral smears, complete blood counts including parameters like haemoglobin level, RBC count, RBC indices, Red cell distribution width, Total WBC count, Packed cell volume, platelet count and these were correlated with the NESTROFT results. In the peripheral smears included in the study, $57 \%$ microcytosis. $26 \%$ of the smears had associated hypochromia. Macrocytosis along with microcytosis was observed in $4 \%$ of smears. One of the smears had features of sicklethalessemia with sickle like cells and microcytic hypochromic RBCs. Associated finding of thrombocytosis was observed in $8 \%$ of the smears.

Out of the 50 patient samples included in the study, 16 samples showed positive results for NESTROFT. Among the NESTROFT positive samples, it was observed that the age range was from 1 year to 23 years. Male: female ratio was 1:3. The mean RBC count was 4.5 millions/cumm. The mean haemoglobin was found to be $9.1 \mathrm{~g} / \mathrm{dl}$. One of the patient's samples, showed a haemoglobin of $13.5 \mathrm{~g} / \mathrm{dl}$ with the peripheral smear showing hypochromia and hence included in this study. The mean MCV was observed to be $66.3 \mathrm{fl}$. Mean MCH was found to be 20.1pg and the Mean MCHC was 30.1\%. The mean RDW-SD in these positive cases was found to be 15.1. Mean Packed cell volume was found to be $30 \%$. The average total white blood cell count was found to be $9.04 \times 10^{9}$. The average platelet count was $3.7 \times 10^{9}$

In the NESTROFT negative group, it was observed that male: female ratio was 1:5. Age range was from one year to 58 years. Mean RBC count was 4.7 millions/cumm. Mean haemoglobin $10 \mathrm{~g} / \mathrm{dl}$. Mean MCV $71 \mathrm{fl}$, mean MCH 22pg and mean MCHC 30.7\%. Average packed cell volume was found to be 33\%. Mean Red cell distribution width-SD was $15.2 \%$. Average total White Blood cell count was $9.2 \times 10^{9}$. Average platelet count was $4 \mathrm{X} 10^{9}$.

The comparison of parameters with the NESTROFT results are shown in the Bar Diagram 1 

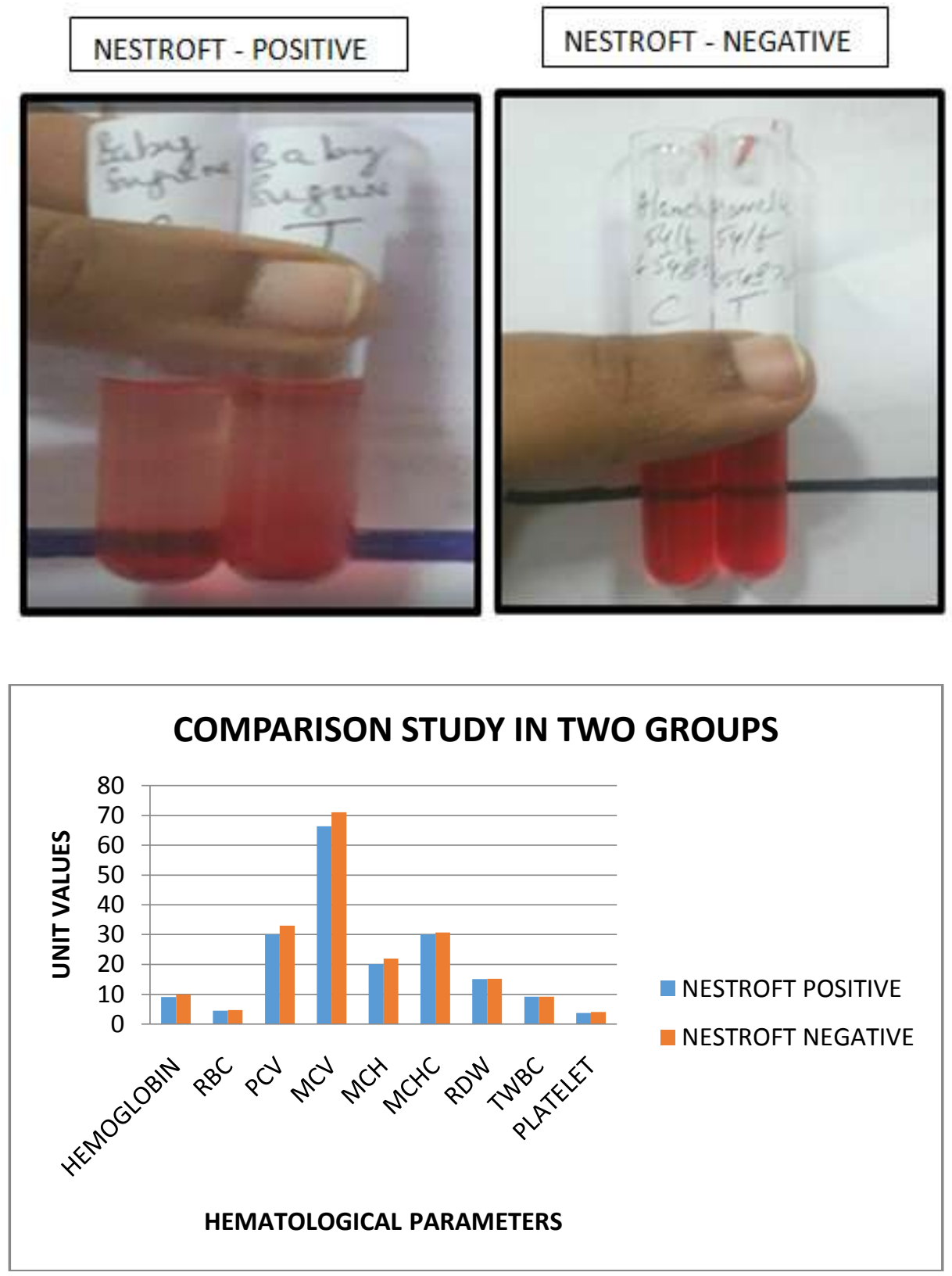

The results of the $\mathrm{P}$ value obtained using Unpaired t test for comparison of the means of all the haematological parameters between the NESTROFT positive and negative groups is shown in the Table 1 .

Table 1: Statistical Significance Of Hematological Parameters With Nestroft

\begin{tabular}{|l|l|l|l|}
\hline Parameter & $\begin{array}{l}\text { Nestroft Positive N=16 } \\
\text { Mean }\end{array}$ & $\begin{array}{l}\text { Nestroft Negative N=34 } \\
\text { Mean }\end{array}$ & P Value \\
\hline Hemoglobin & 9.1 & 10 & 0.9 \\
\hline Rbc & 4.5 & 4.7 & 0.9 \\
\hline Pcv & 30 & 33 & 0.9 \\
\hline Mcv & 66.3 & 71 & 0.9 \\
\hline Mch & 20.1 & 22 & 0.9 \\
\hline Mchc & 30.1 & 30.7 & 0.9 \\
\hline Rdw & 15.1 & 15.2 & 0.9 \\
\hline Twbc & 9200 & 9200 & 0.9 \\
\hline Platelet & 3.7 & 4.0 & 0.9 \\
\hline
\end{tabular}

It was observed that there was no significant difference in all the haematological parameters between the NESTROFT positive group and the negative group. $\mathrm{P}$ value was $>0.05$ for all the parameters. 


\section{Discussion}

Among the hemoglobinopathies, thalassemias have a higher incidence in India. Hence, it becomes necessary for the early detection and diagnosis. Genetic screening, molecular detection methods, high performance liquid chromatography and haemoglobin electrophoresis are the methods available currently for the diagnosis ${ }^{1}$. But, to screen mass populations and as a routine testing in clinical laboratories in underdeveloped countries, a single tube red cell osmotic fragility test was designed. This helps to scrutinize patients who need other investigations like haemoglobin electrophoresis etc. S.K,Bhobate et al have shown in their study that NESTROFT has a sensitivity of $97.1 \%$ and a specificity of $100 \%$. They have observed that NESTROFT was positive in $97 \%$ of known thalassemic trait patients and hence concluded that it is an effective screening test for hemoglobinopathies ${ }^{2}$.

Sanjay Piplani et al have studied the utility of NESTROFT in 150 Indian Punjabi subjects and have concluded that it is a cost-effective, valuable screening test in the rural population ${ }^{3}$.

Giulia Breveglieri et al have studied the Taqman genotyping for detection of single nucleotide polymorphisms frequent in thalassemias ${ }^{4}$. They have observed in their study that these genotyping methods could be used for non-invasive prenatal diagnosis of these disorders. With the availability of these molecular diagnostic methods which can detect Beta globin gene mutations at an early gestational age of 9 weeks, it becomes necessary for screening all the antenatal women for subjecting to futher evaluation.

Manju Mamtani et al have observed in their study that NESTROFT positivity has a marginal significant correlation with elevated $\mathrm{HbA} 2$ levels but none of the haematological parameters correlated with positive NESTROFT ${ }^{5}$. These findings are similar to the observations in our study.

\section{Conclusion}

It is concluded from our study that all peripheral smears with a microcytic hypochromic anaemia especially in women of child bearing age group should be screened for thalassemia trait by using NESTROFT as a routine test in clinical laboratories.

\section{References}

[1]. Dacie, J. V and S. M Lewis. Practical Haematology, 4. Ed. 1st ed. London: J. \& A. Churchill, 1968.

[2]. Bhobate SK, Gaikwad ST, Bhaledrao T. NESTROFT as a screening test for Beta-Thalassemia trait. Indian J Pathol Microbiol; July 2002:45(3); 265-26

[3]. Sanjay Piplani1, Rahul Manan2, Monika Lalit3, Mridu Manjari4, TajinderBhasin5, Jasmine Bawa. NESTROFT - A Valuable, Cost Effective Screening Test for Beta Thalassemia Trait in North Indian Punjabi Population. J Clin Diag Res. Dec 2013; 71(12): 27842787

[4]. Breveglieri G, Travan A, D’Aversa E, Cosenza L, Pellegatti P, Guerra G et al. Postnatal and non-invasive prenatal detection of $\beta$ thalassemia mutations based on Taqman genotyping assays. PLOS ONE. 2017;12(2):e0172756.

[5]. Mamtani M, Das K, Jawahirani A, Rughwani V, Kulkarni H. Is NESTROFT sufficient for mass screening for $\beta$-thalassaemia trait?. Journal of Medical Screening. 2007;14(4):169-173.

[6]. Chakrabarti I, Sinha SK, Ghosh N, Goswami BK. Beta thalassemia carrier detection by NESTROFT: an answer in rural scenario. Iranian J Pathol. 2012; 7(1): 19-26.

[7]. Gomber S, Sanjeev and Madan N. Validity of NESTROFT in screening and diagnosis of beta Thalassemia trait. J Trop Paed. 1997; 43(6): 363-6.

[8]. Maheshwari M, Arora S, Kabra M, Menon PSN. Carrier screening and prenatal diagnosis of beta Thalassemia. Ind Paed. 1999; 36(11): 1119-25. 